\title{
NIELSEN NUMBERS OF PERIODIC MAPS ON SOLVMANIFOLDS
}

\author{
KYUNG BAI LEE
}

(Communicated by Frederick R. Cohen)

\begin{abstract}
Let $f: M \rightarrow M$ be a self-map of a solvmanifold $M$. Then the Lefschetz number $L(f)$ and the Nielsen number $N(f)$ of $f$ satisfy $|L(f)| \leq$ $N(f)$. If $f$ is homotopically periodic (i.e., $f^{k} \simeq$ id, for some $k \geq 1$ ), then $L(f)=N(f)$.
\end{abstract}

Let $M$ be a closed manifold and let $f: M \rightarrow M$ be a continuous map. We study the relations between the two invariants; the Lefschetz number $L(f)$ and the Nielsen number $N(f)$.

These two numbers give information on the existence of fixed point sets. If $L(f) \neq 0$, every self-map $g$ of $M$ homotopic to $f$ has a nonempty fixed point set. The Nielsen number is a lower bound for the number of components of the fixed point set of all maps homotopic to $f$.

Even though $N(f)$ gives more information than $L(f)$ does, it is harder to calculate. For some cases, it is known that they are almost the same.

If $M$ is a torus, then $|L(f)|=N(f)$ [2].

If $M$ is a nilmanifild, then $|L(f)|=N(f)$ [1]

If $M$ is an infranilmanifold and $f$ is homotopically periodic, then $L(f)=$ $N(f)$ [6]. In fact, $|L(f)|=N(f)$ cannot be generalized to infranilmanifolds or solvmanifolds as [6, Theorem 10] shows. In [6] it was also shown that $L(f)=N(f)$ cannot be generalized to nonperiodic maps. In this paper, we show $L(f)=N(f)$ holds for solvmanifolds $M$ if $f$ has finite homotopy period.

Let $S$ be a connected, simply connected solvable Lie group and $H$ be a closed subgroup of $S$. The coset space $H \backslash S$ is called a solvmanifold. We shall talk about compact solvmanifolds only.

Theorem. Let $f: M \rightarrow M$ be a self-map of a solvmanifold $M$. Then $|L(f)| \leq$ $N(f)$. If $f$ is homotopically periodic then $L(f)=N(f)$.

The first part of the statement, which generalizes [1], has been proved in [9]. The second part is a generalization of certain cases of [6]. Note that there are

Received by the editors March 20, 1991.

1991 Mathematics Subject Classification. Primary 55M20; Secondary 55M35.

Key words and phrases. Lefschetz number, Nielsen number, homotopically periodic map, solvmanifold, nilmanifold, flat manifold.

The author was partially supported by the National Science Foundation. 
infranilmanifolds that are not solvmanifolds. The proof uses the same idea as in [9], but many things had to be clarified; especially, the existence of the fully invariant subgroup and the construction of a model space $M^{\prime}$ are explicitly shown. At the final stage, we use the method of $[1,6]$ to get the result.

For a map $f: M \rightarrow M$, define an equivalence relation on $\operatorname{Fix}(f)$ as follows: For $x_{0}, x_{1} \in \operatorname{Fix}(f), x_{0} \sim x_{1}$ if and only if there exists a path $c$ from $x_{0}$ to $x_{1}$ such that $c$ is homotopic to $f \circ c$ relative to the end points. An equivalence class of this relation is called a fixed point class (=FPC) of $f$. Let $p: \widetilde{M} \rightarrow M$ be a universal covering of $M$. It is known that $x_{0} \sim x_{1}$ if and only if $x_{0}, x_{1} \in$ $p \operatorname{Fix}(\tilde{f})$ for some lift $\tilde{f}: \widetilde{M} \rightarrow \widetilde{M}$ of $f$ to the universal covering. Therefore, a fixed point class is of the form $p \operatorname{Fix}(\widetilde{f})$ for some lift $\widetilde{f}: \widetilde{M} \rightarrow \widetilde{M}$. For each fixed point class $C$ of $f$, there is an integer associated with it, called the index of the FPC. Then the fixed point class $C$ is called essential if the index $(f, C)$ is nonzero. See [5, Theorem 1.6].

The rest of this paper is a proof of the theorem. First we construct a new model space $M^{\prime}$ that is homotopy equivalent to $M$ and a map $f^{\prime}: M^{\prime} \rightarrow M^{\prime}$ corresponding to $f$. The space $M^{\prime}$ is a fiber bundle over a torus with a fiber nilmanifold; and $f^{\prime}$ will be fiber-preserving. To use the local product formula for the index of fixed point components, we apply the technique and result of [6, Theorem 2].

Let $\pi=\pi_{1}(M)$ be the fundamental group of $M=H \backslash S$, where $S$ is a connected, simply connected solvable Lie group and $H$ is a closed subgroup. Then $\pi=H / H_{0}$. Such a group $\pi$ is known to be a strongly torsionfree $\mathscr{S}$ group; that is, $\pi$ contains a finitely generated, torsionfree nilpotent normal subgroup $D$ with the quotient $\pi / D$ free abelian of finite rank.

Consider the commutator subgroup $[\pi, \pi]$. Let $F$ be the maximal finite subgroup of the quotient group $\pi /[\pi, \pi]$. Then $F$ is fully invariant (i.e., $F$ is invariant under any endomorphism of $\pi /[\pi, \pi])$. Let $\Gamma$ be the inverse image of $F$ under the quotient map $\pi \rightarrow \pi /[\pi, \pi]$. Then since $[\pi, \pi]$ is fully invariant in $\pi, \Gamma$ is a fully invariant subgroup of $\pi$. We show that $\Gamma$ is nilpotent. Since $\pi / D \cong \mathbb{Z}^{s}$ is torsionfree, the torsion subgroup of $\pi /[\pi, \pi]$ lies in $D /[\pi, \pi]$. This implies that $\Gamma \subset D$, and hence $\Gamma$ is nilpotent. We have found a fully invariant subgroup $\Gamma$ of $\pi$ that is nilpotent and $\pi / \Gamma \cong \mathbb{Z}^{s}$ is free abelian.

With the exact sequence of groups

$$
1 \rightarrow \Gamma \rightarrow \pi \rightarrow \mathbb{Z}^{s} \rightarrow 1
$$

we shall do the Seifert fiber space construction to obtain a bundle over a torus with a nilmanifold as a fiber. It is very important that the fundamental group of the fiber is fully invariant in $\pi$ because the maps involved are not just automorphisms but endomorphisms.

By $\mathrm{Mal}^{\prime} \mathrm{cev}$, there is a unique connected, simply connected nilpotent Lie group $G$ containing $\Gamma$ as a lattice. Let $C\left(\mathbb{R}^{s}, G^{*}\right)$ be the group of all smooth maps of $\mathbb{R}^{s}$ into $G$. The group law is

$$
(\lambda * \eta)(w)=\eta(w) \cdot \lambda(w)
$$

for $\lambda, \eta \in C\left(\mathbb{R}^{s}, G^{*}\right)$ and $w \in \mathbb{R}^{s}$. Let $E(s)=\mathbb{R}^{s} \rtimes O(s)$ be the Euclidean group acting on $\mathbb{R}^{s}$. By choosing a coordinate system on $\mathbb{R}^{s}, E(s)$ is the group of isometries of $\mathbb{R}^{s}$. We can embed our $\mathbb{Z}^{s}$ as standard translations via 
$\rho: \mathbb{Z}^{s} \rightarrow \mathbb{R}^{s} \subset E(s)$. The group $\operatorname{Aut}(G) \times E(s)$ acts on $C\left(\mathbb{R}^{s}, G^{*}\right)$ by

$$
(g, h) \cdot \lambda=g \circ \lambda \circ h^{-1}
$$

for $(g, h) \in \operatorname{Aut}(G) \times E(s)$ and $\lambda \in C\left(\mathbb{R}^{s}, G^{*}\right)$. Now form the semidirect product

$$
\mathscr{U}=C\left(\mathbb{R}^{s}, G^{*}\right) \rtimes(\operatorname{Aut}(G) \times E(s)) .
$$

The group law is

$$
(\lambda, g, h)\left(\lambda_{1}, g_{1}, h_{1}\right)=\left(\lambda * g \lambda_{1} h^{-1}, g g_{1}, h h_{1}\right),
$$

and it acts on $G \times \mathbb{R}^{s}$ by

$$
(\lambda, g, h)(x, w)=(g(x) \cdot \lambda(h(w)), h(w)) .
$$

From the short exact sequence $1 \rightarrow \Gamma \rightarrow \pi \rightarrow \mathbb{Z}^{s} \rightarrow 1$ together with the action of $\mathbb{Z}^{s}$ on $\mathbb{R}^{s}$, we construct a representation of $\pi$ into $\mathscr{U}=C\left(\mathbb{R}^{s}, G^{*}\right) \rtimes$ (Aut $(G) \times E(s))$. The lattice $\Gamma \subset G$ sits in $C\left(\mathbb{R}^{s}, G^{*}\right) \rtimes \operatorname{Inn}(G)$ as left multiplications $(a, \mu(a))$, where $\mu(a)(x)=a x a^{-1}$.

In [7], it is proved that, in this situation, there exists a representation $\pi \rightarrow \mathscr{U}$ so that the following diagram with exact rows is commutative.

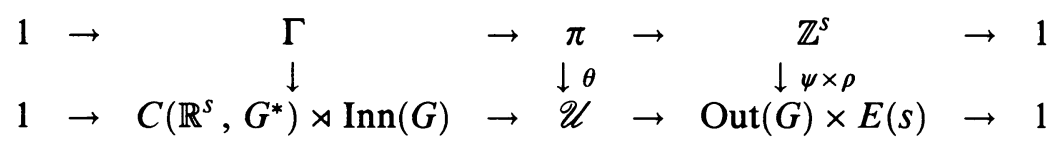

Furthermore, such a $\theta$ is unique up to conjugation by elements of $C\left(\mathbb{R}^{s}, G^{*}\right) \rtimes$ $\operatorname{Inn}(G)$. These are based on the fact that $H^{i}\left(\mathbb{Z}^{s} ; C\left(\mathbb{R}^{s}, G^{*}\right)\right)=0$ for $i=1,2$. These are nonabelian group cohomologies.

The action of $\pi$ on $G \times \mathbb{R}^{s}$ by this representation is properly discontinuous and free because $\pi$ is torsionfree. Thus, the representation of $\pi$ into $\mathscr{U}$ gives rise to a compact smooth manifold $M^{\prime}=\pi \backslash\left(G \times \mathbb{R}^{s}\right)$. The manifold $M^{\prime}$ has a (Seifert) fiber structure

$$
\Gamma \backslash G \rightarrow M^{\prime} \rightarrow \mathbb{R}^{s} / \mathbb{Z}^{s}=T^{s}
$$

with the fiber nilmanifold $N=\Gamma \backslash G$.

Note that $\pi$ is a torsionfree virtually poly $\mathbb{Z}$-group. According to FarrellHsiang, $M$ and $M^{\prime}$ are homeomorphic if $\operatorname{dim} M \neq 3,4$. However, we do not need this fact. We only need the fact that $M$ and $M^{\prime}$ are homotopy equivalent. Let $\alpha: M \rightarrow M^{\prime}$ be a homotopy equivalence with a homotopy inverse $\beta$. Let $f^{\prime}: M^{\prime} \rightarrow M^{\prime}$ be the composite $\alpha \circ f \circ \beta$. Then the homotopy commutative diagram

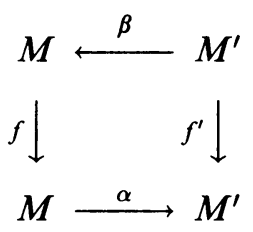

implies that $N(f)=N\left(f^{\prime}\right)$ by the homotopy invariance of the Nielsen number. See [5, Theorem I.5.4]. Of course, $L(f)=L\left(f^{\prime}\right)$ since the Lefschetz number is a homology invariant. 
Thus we shall prove the theorem for $M^{\prime}$ and $f^{\prime}: M^{\prime} \rightarrow M^{\prime}$. For convenience, from now on, we denote $M^{\prime}$ and $f^{\prime}$ by just $M$ and $f$. Therefore our $M$ is the total space of the bundle $\Gamma \backslash G \rightarrow M \rightarrow T^{s}$. Since $\Gamma$ is fully invariant, $f_{\#:}: \pi_{1} M \rightarrow \pi_{1} M$ maps $\Gamma$ into itself.

We argue that there exists a fiber-preserving map $M \rightarrow M$ that is homotopic to $f$. Let $(F, e) \stackrel{i}{\rightarrow}(E, e) \stackrel{p}{\rightarrow}(B, b)$ be a fibration of connected pointed spaces. Assume $B$ is an aspherical space. Let $f: E \rightarrow E$ be a map preserving the base point. Assume that the induced homomorphism $f_{\sharp}: \pi_{1}(E, e) \rightarrow$ $\pi_{1}(E, e)$ leaves the subgroup $(f \circ i)_{\#}\left(\pi_{1}(F, e)\right)$ invariant. When $f \operatorname{did}$ not preserve the base point, one can homotope $f$ so that $f(e)=e$. Note that the homotoping does not change the invariance of the subgroup $f_{\sharp}: \pi_{1}(E, e) \rightarrow$ $\pi_{1}(E, e)$. Then $f_{\#}$ induces a homomorphism $\phi: \pi_{1}(B, b) \rightarrow \pi_{1}(B, b)$ so that $p_{\#} \circ f_{\#}=\phi \circ p_{\#}$. Since $B$ is aspherical, there is a map $g:(B, b) \rightarrow(B, b)$ inducing $\phi$. Then $p \circ f \simeq g \circ p$. By the Covering Homotopy Theorem, there exists $H: E \times I \rightarrow E$ covering the homotopy $p \circ f \simeq g \circ p$ and $H(x, 0)=f(x)$ for all $x \in E$. Now define $f^{\prime}: E \rightarrow E$ by $f^{\prime}(x)=H(x, 1)$. Since $p(H(x, 1))=$ $g(p(x)), f^{\prime}$ is fiber-preserving, and is homotopic to $f$. Note that the spaces $F$ and $E$ need not be aspherical spaces in the argument.

Going back to our model space $M$, we assume that $f$ is fiber-preserving. In fact, when $f_{\#}$ has finite period, such a map $f$ can be found so that it has a lift in the group $\mathscr{U}$.

Let $\bar{f}: T \rightarrow T$ be the induced map on the base space by $f$. If $L(\bar{f})=0$, then $\bar{f}$ is homotopic to a fixed point free map $\bar{f}^{\prime}$. Using the homotopy lifting property of the fibration, we can homotope $f$ to a fixed point free map $f^{\prime}$. Thus, $L(f)=0=N(f)$.

From now on, we assume $L(\bar{f}) \neq 0$. Let $\bar{f}_{*}$ be the endomorphism of $\pi_{1}\left(T^{s}\right)$ induced by $\bar{f}$. Consider $\bar{f}$ as an element of $\mathrm{gl}(s, \mathbb{Z})$. By [2], $\operatorname{det}\left(I-\bar{f}_{*}\right)=L(\bar{f})$, which is nonzero by assumption. This implies that $\bar{f}$ is homotopic to a map with finitely many isolated fixed points. (Observe that the solution of the equation $g(x)=x$ on $\mathbb{R}^{s}$ for an affine map $g(x)=A x+a$, has only one solution if $\operatorname{det}(I-A) \neq 0)$. So let $\operatorname{Fix}(\bar{f})=\left\{b_{1}, b_{2}, \ldots, b_{m}\right\}$. Moreover, all these fixed points have the same index, $\operatorname{sign}(\operatorname{det}(I-A))$, which is either +1 or -1 . Let $\widetilde{f}: \widetilde{M} \rightarrow \widetilde{M}$ be a lift of $f$ to the universal covering and $\widetilde{f}_{\tilde{b}}$ be the restriction of $\widetilde{f}$ to $\widetilde{N}_{\tilde{b}}$, the fiber of $\widetilde{M} \rightarrow \widetilde{T}$ over $\widetilde{b} \in \widetilde{T}$. Note that $\widetilde{N}_{\tilde{b}}$ is a universal cover of $N_{b}$. We shall apply

Theorem [5, IV 3.4, p. 86]. An FPC of $f$ is essential if and only if its projection in $T$ is an essential FPC of $\bar{f}$ and its intersection with an invariant fiber consists of essential fixed point classes of $f_{b}$.

Note that all fixed points $b_{1}, b_{2}, \ldots, b_{m}$ are essential fixed point classes of $\bar{f}$. For each FPC $p \operatorname{Fix}(\tilde{f})$ of $f$, there exists $b \in \operatorname{Fix}(\bar{f})$ such that $p \operatorname{Fix}(\widetilde{f})=$ $p \operatorname{Fix}\left(\widetilde{f}_{\widetilde{b}}\right) \subset N_{b_{i}}$. Moreover, by the above theorem, $p \operatorname{Fix}(\widetilde{f})$ is an essential FPC of $f$ if and only if $p \operatorname{Fix}\left(\tilde{f}_{\tilde{b}}\right)$ is an essential FPC of $f_{b}$. Therefore,

$$
\begin{aligned}
N(f) & =\#(\text { essential FPC of } f)=\sum_{i} \#\left(\text { essential FPC of } f \text { over } b_{i}\right) \\
& =\sum_{i} \#\left(\text { essential FPC of } f_{b_{i}}\right)=\sum_{i} N\left(f_{b_{i}}\right) .
\end{aligned}
$$


On the other hand, we have

$$
\begin{aligned}
L(f) & =\operatorname{index}\left(f, \bigcup_{i} \operatorname{Fix}\left(f_{b_{i}}\right)\right)=\sum_{i} \operatorname{index}\left(f, \operatorname{Fix}\left(f_{b_{i}}\right)\right) \\
& =\sum_{i} \operatorname{index}\left(\bar{f}, b_{i}\right) \cdot \operatorname{index}\left(f_{b_{i}}, \operatorname{Fix}\left(f_{b_{i}}\right)\right)=\sum_{i} \operatorname{index}\left(\bar{f}, b_{i}\right) \cdot L\left(f_{b_{i}}\right) .
\end{aligned}
$$

See [5, IV.3.1, p. 84]. Since index $\left(\bar{f}, b_{i}\right)=+1$ or -1 and is constant for all $i$,

$$
|L(f)|=\left|\sum \pm L\left(f_{b_{i}}\right)\right|=\left|\sum L\left(f_{b_{i}}\right)\right| \leq \sum\left|L\left(f_{b_{i}}\right)\right| .
$$

However, it is known that $\left|L\left(f_{b_{i}}\right)\right|=N\left(f_{b_{i}}\right)$ on a nilmanifold $N_{b_{i}}$ by [1] or [4]. Thus, $|L(f)| \leq \sum_{i}\left|L\left(f_{b_{i}}\right)\right|=\sum N\left(f_{b_{i}}\right)=N(f)$.

Now suppose that $f$ has homotopically finite period. By [6], $L(\bar{f})=N(\bar{f})$. This implies that index $\left(\bar{f}, b_{i}\right)=+1$ for all $i$, and hence $L(f)=\sum_{i} L\left(f_{b_{i}}\right)$. Now $f_{b_{i}}$ has homotopically finite period. Again by [6], $L\left(f_{b_{i}}\right)=N\left(f_{b_{i}}\right)$ for each $i$. Consequently,

$$
L(f)=\sum_{i} L\left(f_{b_{i}}\right)=\sum N\left(f_{b_{i}}\right)=N(f) .
$$

This completes the proof of theorem.

Remarks. To generalize the theorem to infrasolvmanifolds, it seems that one needs to have a product formula for Seifert fiber spaces. Such spaces have singularities, and we do not have a product formula for generalized bundles. The author thanks Professor Frank Raymond for many helpful suggestions.

\section{REFERENCES}

1. D. V. Anosov, The Nielsen numbers of maps of nil-manifolds, Uspekhi. Mat. Nauk 40 (1985), 133-134; English transl., Russian Math. Surveys 40 (1985), 149-150.

2. R. Brooks, R. F. Brown, J. Pak, and D. Taylor, The Nielsen number of maps on tori, Proc. Amer. Math. Soc. 52 (1975), 398-400.

3. R. F. Brown, The Lefschetz fixed point theorem, Scott-Forseman, Chicago, 1971.

4. E. Fadell and S. Husseini, On a theorem of Anosov on Nielsen numbers for nilmanifolds, Nonlinear Functional Analysis and its Applications (Maratea, 1985), NATO Adv. Sci. Inst. Ser. C: Math. Phys. Sci., vol. 173, Reidel, Dordrecht-Boston, MA, 1986, pp. 47-53.

5. B. J. Jiang, Lectures on Nielsen fixed point theory, Contemp. Math., vol. 14, Amer. Math. Soc., Providence, RI, 1983.

6. S. Kwasik and K. B. Lee, The Nielsen numbers of homotopically period maps of infranilmanifolds, J. London Math. Soc. (2) 38 (1988), 544-554.

7. Y. Kamishima, K. B. Lee, and F. Raymond, The Seifert construction and its applications to infranilmanifolds, Quart. J. Math. Oxford Ser. (2) 34 (1983), 433-452.

8. K. B. Lee and F. Raymond, Topological, affine and isometric actions on flat Riemannian manifolds, J. Differential Geom. 16 (1981), 255-269.

9. C. McCord, Nielson numbers and Lefschetz numbers on solvmanifolds, Pacific J. Math. 147 (1991), 153-164. 